\title{
Next-Generation Sequencing in Breast Cancer Management: A Case Report of Genomic Tumour Evolution over Time
}

\author{
Marta Vaz Batista ${ }^{a}$ Diogo Alpuim Costa ${ }^{b, c}$ Paula Borralho ${ }^{c, d}$ \\ Sofia Braga ${ }^{a}, b, c$ \\ ancology Department, Hospital Professor Doutor Fernando Fonseca EPE, Amadora, \\ Portugal; ' bHaematology and Oncology Department, CUF Oncologia, Lisboa, Portugal; \\ 'Pathology Department, CUF Oncologia, Lisbon, Portugal; dNOVA Medical School, \\ Faculdade de Ciências Médicas, Lisbon, Portugal; ${ }^{e}$ Faculdade de Medicina, Universidade de \\ Lisboa, Lisbon, Portugal
}

\section{Keywords}

Case report · Next-generation sequencing · Metastatic breast cancer · Breast cancer in young

\begin{abstract}
The clinicopathological breast cancer subtypes are used in clinical practice to better anticipate biological behaviour and guide systemic treatment strategy. In the adjuvant setting, genomic assay recurrence scores became widely available for luminal-like disease. Recently, nextgeneration sequencing (NGS) platforms have been used, essentially, in more advanced disease setting, in situations refractory to conventional treatment, or even in rare cancers for which there are no established treatment guidelines. Moreover, subpopulations of cancer cells with unique genomes within the same patient may exist across different regions of a tumour or evolve over time, which is called intratumoural heterogeneity. We herein report a case of a 38-year-old woman with breast cancer whose primary and metastatic disease exhibited discordant expression of hormone receptors, with the former being positive and the latter negative. Furthermore, the NGS analysis revealed slight and dynamic changes of mutational profiles between different metastatic lesions, potentially impacting breast cancer management and prognosis. These alterations may reflect tissular and temporal changes in tumour subclones and may also be due to the selective pressure caused by antineoplastic treatment. The use of genomic analyses in order to improve cancer treatment has been studied prospectively with encouraging results. The widespread use of NGS tests in clinical practice also creates new challenges. The most relevant may be to know which genomic alterations detected should be valued and how they should be targeted.
\end{abstract}




\section{Introduction}

Breast cancer (BC) is the most common malignancy and the second cause of cancerspecific death in women from high-income countries [1]. The St. Gallen International Expert Consensus on the Primary Therapy of Early Breast Cancer 2011 recognized considerable progress in the pathological and molecular characterization of breast cancer subtypes. A functional surrogate definition identified by immunohistochemical (IHC) analysis was uniformly adopted to classify tumours as luminal type A or luminal type B based on a combination of oestrogen receptor (ER), progesterone receptor (PgR), and Ki67 status, human epidermal growth factor receptor 2 (HER2) positive or negative, or, by default, triple negative [2]. Biological behaviour and systemic treatment response can be anticipated based on this classification, allowing a more personalized breast cancer management.

In hormone-receptor-positive, HER2-negative early breast cancer, genomic assay recurrence scores provided prognostic information that is independent of clinicopathological features. The addition of this relevant data enabled more precise discrimination of subgroups of women who may derive clinical and meaningful benefit from adding chemotherapy to antioestrogen therapy. The latest American Joint Committee on Cancer (AJCC) TNM staging edition for breast cancer already takes these gene expression panels into account, resulting, in some cases, in downstaging [3].

Recently, next-generation sequencing (NGS) platforms have been used, essentially, in more advanced disease setting, in situations refractory to conventional treatment, or even in rare cancers for which there are no established treatment guidelines [4]. In the era of personalized medicine, whole-genome tumour analysis may lead to detection of targetable molecular alterations, for which specific drugs can be used with expected clinical benefit. Moreover, subpopulations of cancer cells with unique genomes within the same patient may exist across different regions of a tumour or evolve over time, called intratumoural heterogeneity. Therefore, analyses of primary and metastatic tumour samples might be important for selecting the optimal treatment approach.

We herein report a case of a breast cancer patient whose primary and metastatic disease exhibited discordant expression of hormone receptors. Furthermore, the NGS analysis revealed slight and dynamic changes of mutational profiles between different metastatic lesions.

\section{Case Presentation}

A 38-year-old Caucasian premenopausal patient was referred to evaluate a hard, slightly painful lump in the lower outer quadrant of the left breast with no associated fever, nipple discharge, or skin redness. She reported 2 months before a history of local breast trauma. Besides smoking habits (20 pack-year), she had no relevant past medical history. Family history included mother and great aunt previously diagnosed with breast cancer over the age of 65 years.

The patient underwent an ultrasound-guided core biopsy, and the histopathological examination revealed an invasive no special type carcinoma, poorly differentiated (G3) and luminal type B (ER 30\%, PgR negative, HER2 negative, and Ki67 98.4\%) (Fig. 1). Laboratory data indicated no abnormalities, including serum levels of CEA and CA15-3. Following proper diagnostic and staging workup, the patient had undergone breast conserving surgery with a pathological classification of pT2(35 mm)N0(sn)M0 (stage II AJCC). After a multidisciplinary tumour board, adjuvant chemotherapy ( 4 cycles of doxorubicin $60 \mathrm{mg} / \mathrm{m}^{2}$

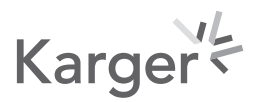




\section{Case Reports in Oncology}
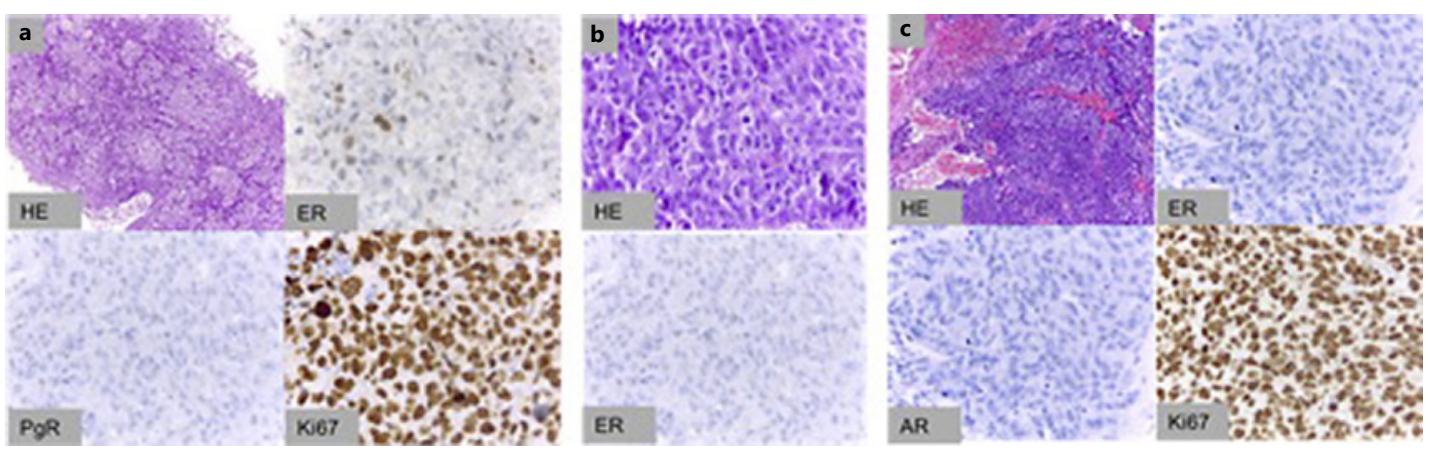

Fig. 1. Immunohistochemical analysis: primary breast tumour (invasive carcinoma no special type, G3, ER 30\%, PgR negative, HER2 negative, and Ki67 98.4\%) (a); adrenal metastasis (ER 0\%, PgR 1\%, HER2 negative, and Ki67 > 90\%) (b); brain metastasis (triple negative, androgen receptor negative, and Ki67 >90\%) (c). $\mathrm{AR}$, androgen receptor; ER, oestrogen receptor; G3, poorly differentiated; HE, haematoxylin and eosin; HER2, human epidermal growth factor receptor 2; PgR, progesterone receptor.

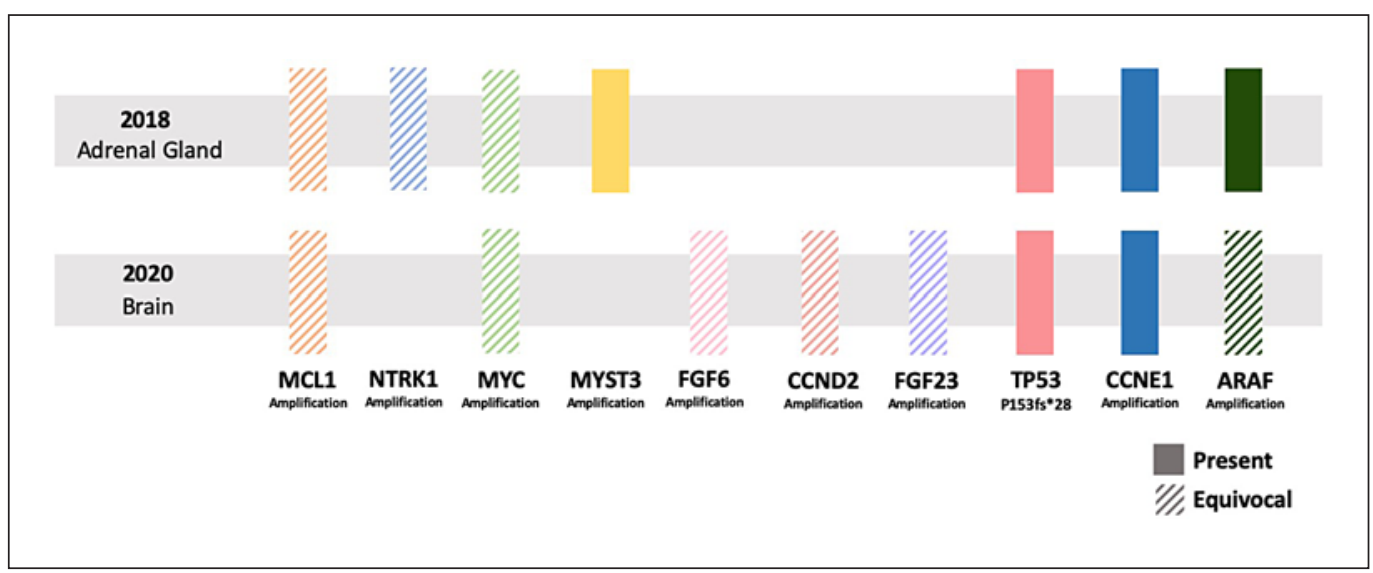

Fig. 2. Genomic alterations found using the F1CDx test: superior line showing alterations found on adrenal gland metastasis in 2018, and inferior line showing alterations found on brain metastasis in 2020. Gene amplifications on MCL1, NTRK1, MYC, FGF6, CCND2, FGF23, and ARAF (brain metastasis) were reported as equivocal as their copy numbers are estimated as 7. ARAF, serine/threonine-protein kinase A-Raf; CCN, cyclin; FGF, fibroblast growth factor; F1CDx, FoundationOne ${ }^{\circledR}$ CDx; MCL, myeloid cell leukaemia; MYC, MYC protooncogene, BHLH transcription factor; MYST, Moz, YBF2, Sas2p, Tip; NTRK, neurotrophic receptor tyrosine kinase; TP, tumour protein.

and cyclophosphamide $600 \mathrm{mg} / \mathrm{m}^{2}$ every 3 weeks followed by 12 weeks of paclitaxel 80 $\mathrm{mg} / \mathrm{m}^{2}$ ) and radiotherapy ( $60 \mathrm{~Gy}$ ) were indicated. Adjuvant hormonotherapy was postponed due to the patient's desire to get pregnant a second time. Approximately 20 months after breastsurgery, the disease progressed with asymptomatic bilaterallung micronodules and a haemorrhagic nodular lesion $(32 \times 41 \mathrm{~mm})$ of the left adrenal gland. The patient was submitted to emergency left adrenalectomy for retroperitoneal haematoma control. The surgical specimen confirmed adrenal metastasis, with IHC slightly different from the primary tumour, with ER negative and PgR 1\%, and the remainder being similar (HER2 negative, G3, and Ki67 > 90\%) (Fig. 1). Serum tumour markers remained within the normal range. NGS with FoundationOne ${ }^{\circledR} \mathrm{CDx}(\mathrm{F} 1 \mathrm{CDx})$ was performed on metastatic adrenal gland tissue (Fig. 2). In addition, programmed death-ligand 1 expression was negative. On F1CDx, the tumour was microsatellite stable, and tumour mutation burden 


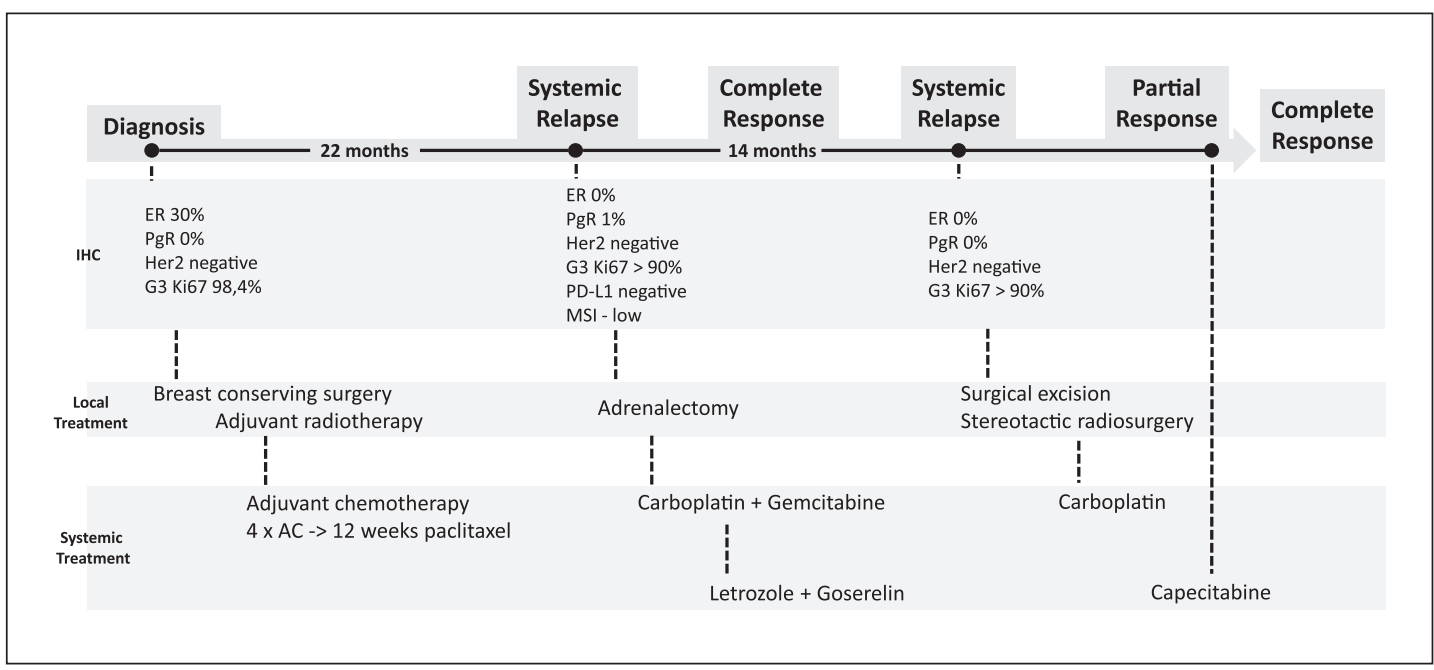

Fig. 3. Clinical evolution since diagnosis, including immunohistochemical findings and local and systemic treatments. F1CDx was performed by the time of the first (adrenal gland) and second (brain metastasis) systemic relapses, 22 and 36 months after diagnosis, respectively. AC, doxorubicin combined with cyclophosphamide; ER, oestrogen receptor; F1CDx, FoundationOne ${ }^{\circledR} \mathrm{CDx}$; G3, poorly differentiated; HE, haematoxylin and eosin; HER2, human epidermal growth factor receptor 2; PgR, progesterone receptor.

was low (4 mutations/megabase). No actionable targets for approved drugs in breast cancer were found. An early control positron emission tomography-computed tomography (PET-CT) revealed 5 hypermetabolic lesions: 3 lymph nodes in the posterior mediastinum, 1 in the adrenal gland bed, and 1 in the right iliac crest. The patient was treated with 6 cycles of carboplatin (AUC5) and gemcitabine $\left(1,000 \mathrm{mg} / \mathrm{m}^{2}\right)$ with dose reductions and delays due to pancytopenia (G2 anaemia, G2 thrombocytopaenia, and G3 neutropenia; Common Terminology Criteria for Adverse Events version 5.0; CTCAE v5.0). Complete response was confirmed both in CT and in PET-CT. Despite the favourable clinical benefit, the treatment was switched to maintenance endocrine therapy with letrozole $2.5 \mathrm{mg}$ daily and goserelin $3.6 \mathrm{mg}$ once a month. However, 14 months later, she experienced incoordination of the left upper limb and hemineglect with 2 days of evolution. Brain magnetic resonance (MRI) showed a single right parietal lesion $(35 \mathrm{~mm}$ ). Complete surgical excision was performed and stereotactic radiosurgery given to surgical bed (central dose: 37 Gy; peripheral dose: 18.5 Gy). Pathological analysis confirmed to be a metastatic lesion, triple negative, androgen receptor negative, and with a high proliferative index (Fig. 1). NGS using F1CDx was performed again (Fig. 2). Still, no actionable target was found, but a different genomic profile landscape was identified. The tumour was microsatellite stable and tumour mutation burden low (5 mutations/megabase). PET-CT also revealed new hypermetabolic lesions: 1 in the upper lobe of right lung, 1 lymph node in the right paratracheal lymph region, and 1 in the left iliac crest. She was planned to receive 6 additional cycles of carboplatin AUC6, but only 5 were actually administered due to G3 haematological toxicity (CTCAE v5.0). Re-evaluation with PET-CT revealed partial response, reducing lesions in the upper lobe of the right lung lobe and right paratracheal lymph node and disappearance of lung micronodules and iliac crest lesions. Brain MRI re-evaluation was without new lesions. Thereafter, she was administered capecitabine 1,250 $\mathrm{mg} / \mathrm{m}^{2}$ (twice daily for 14 days every 3 weeks) for 6 cycles with metabolic complete response. The patient has been followed up regularly with clinical examination and imaging. Figure 3 shows clinical evolution. 


\section{Discussion/Conclusion}

Cancer progression is complex, involving clonal expansion, genetic diversification, and clonal selection [5]. The Darwinian evolution of cancer during treatment may explain the positive selection of cells and how drug resistance develops. Our case report exemplifies this biological and molecular dynamic process.

The natural history of cancer is characterized by periods of varying stability with potential intercurrent crises when disease relapses or progresses [6]. During treatment, the population of clones is selected, and the number of genomic mutations is reported to be less at the metastatic site compared to the primary tumour. In this case, there were pathological features suggesting tumour heterogeneity.

First of all, there was a discrepancy in the ER expression between primary tumour (ER positive) and metastasis (both ER negative). However, this is not an unusual finding, with a previous meta-analysis showing pooled discordance proportions of up to $20 \%$ for ER, more frequently changing from ER positive to ER negative [7]. Interestingly, the first metastasis (adrenal gland) expressed PgR 1\% and later on, after 2 consecutive treatment lines with chemotherapy and hormonotherapy, the second biopsied metastasis no longer expressed hormone receptors (including androgen receptor). Recent data suggested that HER2-negative breast cancer with "weak" ER/PgR expression levels by IHC analysis should be considered as the triple-negative subtype. International guidelines recommended that IHC staining of $\geq 1 \%$ for ER and PgR should be considered positive. Furthermore, the decision regarding hormonotherapy in these patients should be made after weighing risks and benefits. As a complete response was achieved with an induction chemotherapy regimen in our patient, it was decided to maintain active treatment with an aromatase inhibitor combined with a $\mathrm{GnRH}$ analogue. This multimodal treatment approach, which included an initial polychemotherapy regimen to control visceral disease followed by maintenance hormone therapy, allowed better management of treatment-induced toxicity and a progression-free interval of $>2$ years.

We also found some discrepancies at genomic level between metastasis obtained from different samples in different time points, with 2 years of interval (adrenal gland and parietal lobe). Genomic analysis was performed with the F1CDx Cancer Panel, which consisted of 324 selected genes and introns of 36 genes involved in rearrangements. Seven genomic alterations were found in adrenal metastasis and 8 in brain metastasis. Five of these genes matched. As mentioned earlier, these alterations may reflect tissular and temporal changes in tumour subclones and may also be due to the selective pressure caused by antineoplastic treatment. In fact, after the first relapse, the patient was treated with a platin doublet for 6 cycles and approximately 12 months with endocrine therapy. Of note, genomic analysis of the adrenal gland showed MYST3 and NTRK1 amplifications that were not present at brain metastasis. On the other hand, the latter showed CCND2, FGF23, and FGF6 amplifications that were not present before. Interestingly, CCND2, FGF23, and FGF6 are colocalized at chromosomal locus $12 \mathrm{p} 13$ and reported to be coamplified together [8], as is the case here. Some stable genomic alterations were also found: TP53 frameshift insertion P153fs*28, ARAF, MYC, CCNE1, and MCL1 amplifications. Table 1 shows, for each genomic alteration identified, the biological relevance of the gene, frequency of the alteration in invasive breast cancer according to The Cancer Genome Atlas [9], and potential clinical significance.

The use of genomic analyses in order to improve cancer treatment has been studied prospectively with encouraging results, either in trials including different types of cancer (MOSCAT0-01) [10] or specifically in breast cancer metastatic cancer (SAFIR01/UNICANCER) [11]. A retrospective analysis of metastatic breast cancer with whole-exome sequencing identified 12 genes significantly mutated, including TP53 [12], an earlier genomic alteration that we also found.

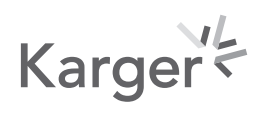


Batista et al.: Next-Generation Sequencing in Breast Cancer Management

Table 1. Genomic alterations identified on F1CDx analysis, biological relevance of the gene, relative frequency in breast cancer, and potential clinical significance

\begin{tabular}{|c|c|c|c|}
\hline $\begin{array}{l}\text { Genomic alteration } \\
\text { identified }\end{array}$ & $\begin{array}{l}\text { Biological relevance of } \\
\text { the gene }\end{array}$ & $\begin{array}{l}\text { Frequency in } \\
\text { breast cancer, } \%\end{array}$ & Potential clinical significance \\
\hline ARAF amplification & $\begin{array}{l}\text { Encodes a member of } \\
\text { MEK-ERK signalling } \\
\text { cascade }\end{array}$ & $0-1$ & $\begin{array}{l}\text { RAF kinase might be targetable by } \\
\text { pan-kinase inhibitors }\end{array}$ \\
\hline MYC amplification & $\begin{array}{l}\text { Encodes a transcription } \\
\text { factor related to cell } \\
\text { cycle regulation }\end{array}$ & 15 & $\begin{array}{l}\text { No direct targeted therapy available; } \\
\text { synthetic lethal strategies might be } \\
\text { useful in future; might predict } \\
\text { response to chemotherapy }\end{array}$ \\
\hline CCNE1 amplification & Encodes cyclin E1 & 2.80 & $\begin{array}{l}\text { No direct targeted therapy available; } \\
\text { might predict resistance to CDK } 4 / 6 \\
\text { inhibitors, amplification might be } \\
\text { correlated with poor prognosis }\end{array}$ \\
\hline MCL1 amplification & $\begin{array}{l}\text { Encodes a member } \\
\text { of BCL2 }\end{array}$ & 15 & $\begin{array}{l}\text { No direct targeted therapy available } \\
\text { yet; might confer resistance to } \\
\text { antitubulin drugs }\end{array}$ \\
\hline MYST3 amplification & $\begin{array}{l}\text { Encodes a protein that } \\
\text { interferes with } \\
\text { transcription factors }\end{array}$ & Low & No direct targeted therapy available \\
\hline NTRK1 amplification & $\begin{array}{l}\text { Encodes a protein that } \\
\text { activates downstream } \\
\text { signalling pathways }\end{array}$ & 4.60 & $\begin{array}{l}\text { NTRK1 fusions genes might be } \\
\text { targetable with TRK inhibitors; no } \\
\text { data on NTRK1 amplifications }\end{array}$ \\
\hline CCND2 amplification & $\begin{array}{l}\text { Encodes a protein } \\
\text { responsible for cell } \\
\text { cycle control }\end{array}$ & 1.5 & $\begin{array}{l}\text { Unknown if amplification or } \\
\text { mutation predicts response to } \\
\text { CDK4/6 inhibitors }\end{array}$ \\
\hline FGF23 amplification & $\begin{array}{l}\text { Encodes a member of } \\
\text { fibroblast growth factor } \\
\text { protein family }\end{array}$ & 1.30 & $\begin{array}{l}\text { Limited data suggest a potential role } \\
\text { for pan-FGFR inhibitors }\end{array}$ \\
\hline FGF6 amplification & $\begin{array}{l}\text { Encodes a member of } \\
\text { fibroblast growth factor } \\
\text { protein family }\end{array}$ & 1.30 & $\begin{array}{l}\text { Limited data suggest a potential role } \\
\text { for pan-FGFR inhibitors }\end{array}$ \\
\hline TP53 P153fs*28 & Tumour suppressor gene & $27-37$ & No direct targeted therapy available \\
\hline
\end{tabular}

ARAF, serine/threonine-protein kinase A-Raf; CCN, cyclin; CDK4/6, cyclin-dependent kinase 4/6; FGF, fibroblast growth factor; F1CDx, FoundationOne® CDx; MCL, myeloid cell leukaemia; MYC, MYC protooncogene, BHLH transcription factor; MYST, Moz, YBF2, Sas2p, Tip; NTRK, neurotrophic receptor tyrosine kinase; TP, tumour protein; TRK, tropomyosin receptor kinase.

The widespread use of NGS tests in clinical practice also creates new challenges. The most relevant may be to know which genomic alterations detected should be valued and how they should be targeted. Major clinical oncology societies have already recognized this unmet clinical need and made appropriate recommendations. One example is the ESMO Scale for Clinical Actionability of molecular Targets (ESCAT) [13], which ranks the level of evidence of individual recurrent genomic alterations to help prioritize treatment. In this case report, however, no targetable mutation from tiers I or II was found, so no targeted therapy was pursued. The low mutational burden and absence of microsatellite instability discouraged the use of immunotherapy. Recently, it was reported that tumours that benefit the most from immune checkpoint inhibitors have microsatellite instability and/or higher tumour mutational burden [14]. Finally, integrative medicine should take into account tissue genomic alterations, 
gene sequencing of cell-free DNA, and human microbiome to give new and improved insights into the clinicopathological picture [15].

Further integrative and comprehensive studies are needed and should include analysis of multiple intersecting dimensions of genomic data. These findings should always be analysed taking into account clinical outcomes to assess the translational impact of these findings in "real-world" practice.

\section{Acknowledgment}

The authors thank CUF Oncologia for all the support.

\section{Statement of Ethics}

The patient gave written informed consent for publication of the case report and any accompanying images. The study is exempt from ethics committee approval since it is a case report.

\section{Conflict of Interest Statement}

The authors have no conflicts of interest to declare.

\section{Funding Sources}

No funding was received.

\section{Author Contributions}

All authors contributed to design and implementation of the case report. Marta Vaz Batista and Diogo Alpuim Costa wrote the manuscript. Paula Borralho and Sofia Braga supervised the work.

\section{Data Availability Statement}

The data that support the findings of this study are available from the corresponding author, upon reasonable request.

\section{References}

1 Sung H, Ferlay J, Siegel RL, Laversanne M, Soerjomataram I, Jemal A, et al. Global cancer statistics 2020: GLOBOCAN estimates of incidence and mortality worldwide for 36 cancers in 185 countries. CA Cancer J Clin. 2021;71:1-41.

2 Goldhirsch A, Wood WC, Coates AS, Gelberg RD, Thurlimann B, Senn HJ, et al. Strategies for subtypes-dealing with the diversity of breast cancer: highlights of the St. Gallen International Expert Consensus on the Primary Therapy of Early Breast Cancer. Ann Oncol. 2011;22:1736-47.

3 Giuliano AE, Connolly JL, Edge SB, Mittendorf EA, Rugo HS, Solin LJ, et al. Breast cancer-major changes in the American Joint Committee on Cancer eighth edition cancer staging manual. CA Cancer J Clin. 2017;67:290-303. 
4 Su Z, Ning B, Fang H, Hong H, Perkins R, Tong W, et al. Next-generation sequencing and its applications in molecular diagnostics. Expert Rev Mol Diagn. 2011;11(3):333-43.

5 Greaves M, Maley CC. Clonal evolution in cancer. Nature. 2012;481:306-13.

6 Torres L, Ribeiro FR, Pandis N, Andersen JA, Heim S, Teixeira MR, et al. Intratumor genomic heterogeneity in breast cancer with clonal divergence between primary carcinomas and lymph node metastases. Breast Cancer Res Treat. 2007;102:143-55.

7 Aurilio G, Disalvatore D, Pruneri G, Bagnardi V, Viale G, Curigliano G, et al. A meta-analysis of oestrogen receptor, progesterone receptor and human epidermal growth factor receptor 2 discordance between primary breast cancer and metastases. Eur J Cancer. 2014;50(2):277-89.

8 Parish A, Schwaederle M, Daniels G, Piccioni D, Fanta P, Schwab R, et al. Fibroblast growth factor family aberrations in cancers: clinical and molecular characteristics. Cell Cycle. 2015;14(13):2121-8.

9 The Cancer Genome Atlas Network. Comprehensive molecular portraits of human breast tumours. Nature. 2012;490:61-70.

10 Massard C, Michiels S, Ferté C, Le Deley MC, Lacroix L, Hollebecque A, et al. High-throughput genomics and clinical outcome in hard-to-treat advanced cancers: results of the MOSCATO 01 trial. Cancer Discov. 2017; 7(6):586-95.

11 André F, Bachelot T, Commo F, Campone M, Arnedos M, Dieras V, et al. Comparative genomic hybridisation array and DNA sequencing to direct treatment of metastatic breast cancer: a multicentre, prospective trial (SAFIR01/UNICANCER). Lancet Oncol. 2014;15:267-74.

12 Lefebvre C, Bachelot T, Filleron T, Pedrero M, Campone M, Soria JC, et al. Mutational profile of metastatic breast cancers: a retrospective analysis. PLos Med. 2016;13(12):e1002201.

13 Mateo J, Chakravarty D, Dienstmann R, Jezdic S, Gonzalez-Perez A, Lopez-Bigas N, et al. A framework to rank genomic alterations as targets for cancer precision medicine: the ESMO Scale for Clinical Actionability of molecular Targets (ESCAT). Ann Oncol. 2018;29:1895-902.

14 Samstein RM, Lee CH, Shoushtari AN, Hellmann MD, Shen R, Janjigian YY, et al. Tumor mutational load predicts survival after immunotherapy across multiple cancer types. Nat Genet. 2019;51:202-6.

15 Alpuim DC, Nobre JG, Batista MV, Ribeiro C, Calle C, Cortes A, et al. Human microbiota and breast cancer: is there any relevant link? A literature review and new horizons toward personalised medicine. Front Microbiol. 2021; 12:584322. 